\title{
TRANSCRIPTOME ANALYSIS TO CHARACTERIZE GENES RELATED TO THE MARBLING TRAIT ON MEAT QUALITY OF ABERDEEN ANGUS
}

\author{
X. WANG, Y. ZHANG*, D. WANG, G, Jin, B.Li, F. XU AND J. CHENG \\ Institute of Animal Husbandry and Veterinary \\ Shanxi Academy of Agricultural Sciences \\ Taiyuan, Shanxi, 030 032, China
}

We compared the intramuscular fat deposition in ribeye and subcutaneous fat muscle of Aberdeen Angus beef cattle. Eight samples of the best and worst muscle marbling grade of Angus cattle were collected from 120 Angus cattle after slaughtering. High-quality RNA was subsequently extracted from the beef ribeye and subcutaneous fat muscle and the transcriptome was sequenced using RNAseq technology. Approximately 37.22 GByte of clean sequencing reads were obtained in this study. A total of 15 differentially expressed genes (DEGs) and 16 DEGs were detected in the ribeye and subcutaneous fat muscle tissues respectively. In the beef ribeye, six genes were up-regulated (CYP1A1, RRAD, RETRG1, LOC787269, XIRP1, OTUD1) and nine genes down-regulated (Bos_taurus_newGene_1359, Bos_taurus_newGene_4387, KRT18, MYL9, ACTA2, PTGDS, DSTN, CD5L, MYL6B), whereas the subcutaneous fat muscle, characterized by good marble meat quality, had 14 genes up-regulated (CYP1A1, SLC25A13, S100A1, KLHL40, THRSP, MYLK4, TNFRSF12A, RRAD, HMOX1, AMPD3, DNAJA4, PTPN3, LOC782922, IFITM10) and two down-regulated genes (Bos_taurus_newGene_996, FST). Gene analysis revealed that the differentially expressed genes were mostly associated with myocyte differentiation, for example MYL9, ACTA2 and MYLK4 genes. Gene ontology (GO) was then used to perform a term enrichment analysis and significant GO terms included the vascular smooth muscle contraction pathway and arachidonic acid (AA) metabolism. Taken together, we observed that the DEGs play an important role in marble texture and intramuscular fat deposition of the different parts of Angus cattle. Hence, a strong basis for improving the meat quality of Aberdeen Angus was considered.

Key words: Angus, DEGs, Intramuscular fat content, Marbling grade, Transcriptome

Beef cattle have become a vital, daily food source for human consumption (Zuo, 2014), because of its high protein, low fat, low cholesterol and other characteristics (Chen, 2015). The quality of beef is dependent on a number of factors, previously identified to include meat color, tenderness, hydraulic and intramuscular fat (IMF) content. The difference between intra- and inter-muscular fat depends on the distribution of the fat. Intermuscular fat is when fat is distributed in adipocytes

\footnotetext{
*Corresponding Author
} 
between the muscle fibers to form marbletextured muscle meat, whereas intramuscular fat is when fat droplets are formed and distributed within the muscle fibers. Their content and fiber structure are essential to the quality, palatability, appearance and nutritional value of the beef, and thus an important determinant in the decision quality index (Hwang et al., 2010; Scollan et al., 2006). Nevertheless, differences between cattle breeds, growth rates, perception and the expected quality exist. The Angus cattle are native to the northeast of Scotland and well known for their good meat, easy growth, coarse feeding and large muscle-stone texture. It is arguably one of best beef varieties in the world (Li, 2002). For the cattle, ribeye and subcutaneous fat muscle are the main parts of intramuscular fat deposition and play the important roles in stabilizing the metabolic balance of the body.

In recent years, the rapid development of sequencing technology has made RNAsequencing a mainstream and cost-effective method for the quantification of gene expression (Li et al., 2015). The Illumina HiSeq TM 2500 sequencing platform adopts the principle of reversible end-side synthesis, which makes it superior to traditional genomics and functional genomics on the account of higher flux, higher sensitivity and higher accuracy (Hou, 2011). Such transcriptome analyses evaluate the sum of all the transcription products in a certain physiological condition and are used to study phenotypes and gene functions (Blencowe et al., 2009). Previously, high-throughput sequencing technologies have highlighted differences in the transcriptome of different muscle types. It demonstrated the importance of increasing our understanding and the significance of studies exploring the relationship between gene expression and the phenotypic mechanisms of IMF (Aksyonov et al., 2006).

The distribution of beef muscle and IMF sedimentation is dependent on the variety, the individual, nutrition level etc (Pethick et al., 2004). The marble texture is regulated by multiple genes, but the IMF content cannot direct detection in living (Grobet $e t$ $a l .$, 1997). Research into the gene(s) and molecular mechanism(s) of IMF deposition has, therefore, become a focus point when looking at beef quality. The study presented herein used an Illumina HiSeq TM 2500 sequencing platform to compare the gene expression in different marbled muscle textures of female Angus cattle and identify differentially expressed genes. Following their functional annotation and a Kyoto Encyclopedia of Genes and Genomes (KEGG) pathway analysis, these genes were associated with muscle or muscle fat and used to elucidate the underlying molecular mechanism of fatty acid deposition in different parts of the body.

\section{MATERIALS AND METHODS}

All experimental animals were 1.5 years old and obtained from the Animal Science and Veterinary Medicine of Shanxi Agricultural 
College. Within 30 minutes of slaughter, four female cattle were selected from 120 Angus cattle after slaughtering and $5 \mathrm{~cm}^{3}$ samples of beef ribeye and subcutaneous fat muscle of the highest and lowest marble level were taken. There were two biological replicates of each cut (eight samples per animal), which were all stored in liquid nitrogen until use. Beef ribeye was labeled $\mathrm{Y}$ and subcutaneous fat muscle as ZP.

RNA extraction and quantification: Total RNA of the muscle tissues was extracted using Trizol (Invitrogen, Carlsbad, USA) following the manufacturer's protocol. RNA concentrations were measured with the Qubit RNA broad-range Assay Kit and a Qubit 2.0 Fluorometer (Life Technologies, California, USA). RNA integrity was assessed using the RNA Nano 6000 Assay Kit and the Agilent Bioanalyzer 2100 system (Agilent Technologies, CA, USA).

Transcriptome library preparation and sequencing: After total RNA qualification and quantification, $1 \mu \mathrm{g}$ of RNA per sample was used for the library construction (BMK cloud platform). Total mRNA samples were first obtained using poly-T oligo-attached magnetic beads (dT) and then fragmented using divalent cations. These fragments were then used to obtain a cRNA library by (1) reverse transcription of the template, (2) phosphorylation, (3) addition of a sequence connector, (4) enrichment of the fragments, and finally, (5) purification by PCR amplification. Finally, the cRNA library was performed using paired-end sequencing. Sequence reads containing adapters, poly- $\mathrm{N}$ and low-quality reads were removed before performing the downstream bioinformatic analyses. Cufflinks was used to map the clean data to the reference genome of Bos taurus. Based on these alignments, the genes were annotated and also new genes were discovered, which were submitted to Gene Bank. A quantitative analysis of the gene expression levels identified the differentially expressed genes and SPSS was used to determine their statistical significance. KEGG pathway analyses were performed using DAVID.

\section{RESULTS}

After cleaning, the RNA-seq data set was reduced to 37.22 GByte for further analysis. The clean reads ranged from 20824314 to 35324449 and the guanine-cytosine content (GC-content) between $48.72 \%$ and $50.04 \%$. The quality score of 30 to clean bases was more than $96.69 \%$ for all eight samples, which is indicative of the high quality of our sequencing data (Table 1).

Identification of differentially expressed genes: We obtained the distribution of fragments per kilobase of transcript per million mapped reads (FPKM):

$$
F P K M=\frac{c D N A \text { Fragments }}{\text { Mapped Fragments }(\text { Millions }) \times \text { Transcript Length }(k b)}
$$


The protein-coding gene expression level of magnitude across $10^{\wedge} 2$ to $10^{\wedge} 4$ in the FPKM values was sequenced six orders eight samples (Fig. 1).

Table 1. Sequence data statistics

\begin{tabular}{lcccc}
\hline Samples & Clean reads & Clean bases & GC Content $(\%)$ & $\geq \mathbf{Q 3 0}$ \\
\hline Y11 & $28,305,026$ & $4,729,548,532$ & 49.90 & 96.92 \\
Y12 & $24,894,792$ & $4,163,607,540$ & 50.04 & 96.92 \\
Y21 & $26,300,616$ & $4,413,944,094$ & 49.60 & 96.98 \\
Y22 & $33,518,491$ & $5,617,008,512$ & 49.57 & 96.96 \\
ZP11 & $35,324,449$ & $5,903,105,288$ & 49.17 & 96.93 \\
ZP12 & $31,234,110$ & $5,249,996,185$ & 48.96 & 97.00 \\
ZP21 & $22,251,594$ & $3,676,074,808$ & 49.39 & 96.69 \\
ZP22 & $20,824,314$ & $3,462,843,357$ & 48.72 & 96.79 \\
\hline
\end{tabular}

Y: Beef ribeye, ZP: Subcutaneous fat muscle, Q30: Quality s

Table 2. List of abbreviations

\begin{tabular}{lll}
\hline Number & Abbreviation & The full name \\
\hline 1 & DEGs & Differentially expressed genes \\
2 & GO & Gene ontology \\
3 & IMF & Intramuscular fat \\
4 & KEGG & Kyoto Encyclopedia of Genes and Genomes \\
5 & GC-content & Guanine-cytosine content \\
6 & FPKM & Fragments per kilobaseof exon per million fragments mapped \\
7 & CYP1A1 & Cytochrome P450, family 1, subfamily A, polypeptide 1 \\
8 & RRAD & Ras-related Associated with Diabetes \\
9 & CYP21A2 & Cytochrome P450, family 21, subfamily A, polypeptide 2 \\
10 & E2F & Dimerization partner 1 \\
11 & DP1 & Dimerization partner \\
12 & KHLH40 & Kelch-like family member 40 \\
13 & THRSP & Thyroid hormone response protein \\
14 & RORC & ROR-gamma \\
15 & MYL9 & Myosin light chain 9 \\
16 & ACTA2 & $\alpha-2$ actin \\
17 & MYLK4 & Myosin light chain kinase 4 \\
18 & AA & Arachidonic acid \\
\hline
\end{tabular}


Indian Journal of Animal Health, December, 2018

Transcriptome analysis of the marbling trait

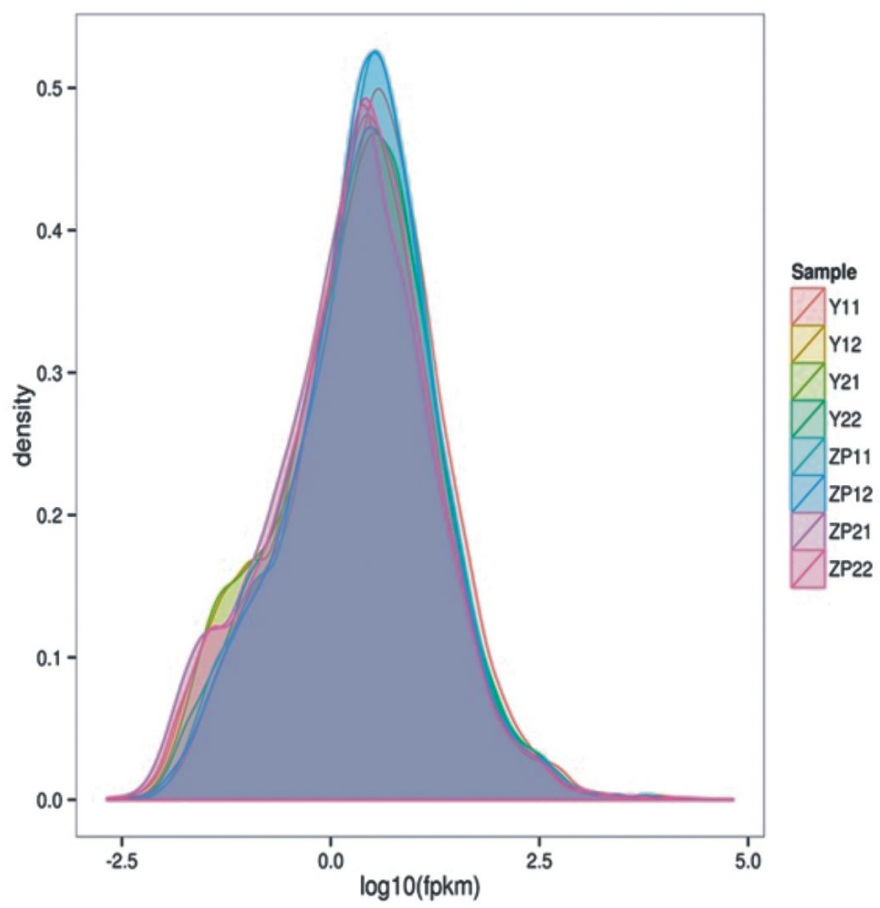

Fig. 1. Comparison of FPKM density distribution of each sample

(Figure in the curve of the different colors represent different samples, the horizontal axis of pointsin the curve corresponding to the sample for numerical FPKM, point ordinate said probability density.)

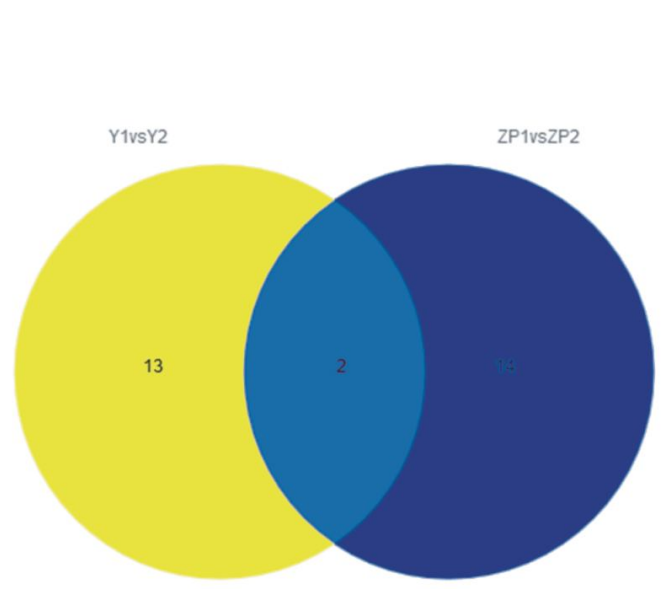

Fig. 2. Venn of the differential gene of y11-y12 vs y21-y22 and zp11-zp12 vs zp21-zp22

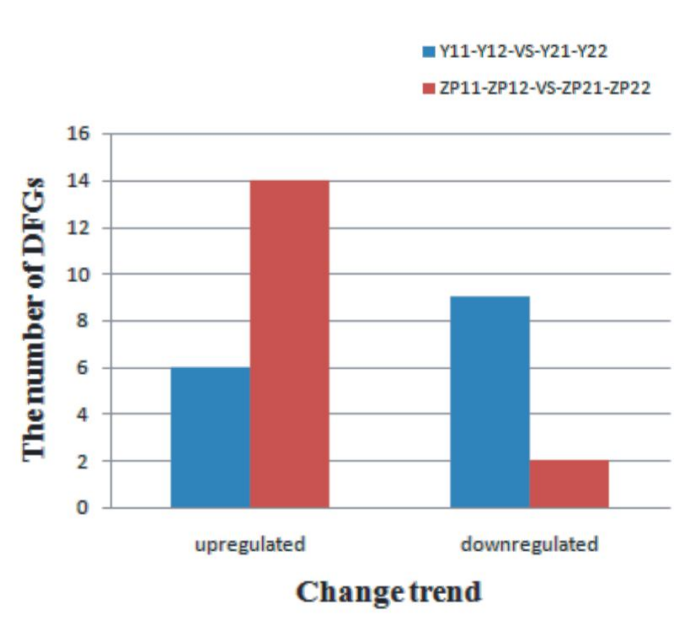

Fig. 3. Significant differentially expressed genes 
Indian Journal of Animal Health, December, 2018

Transcriptome analysis of the marbling trait

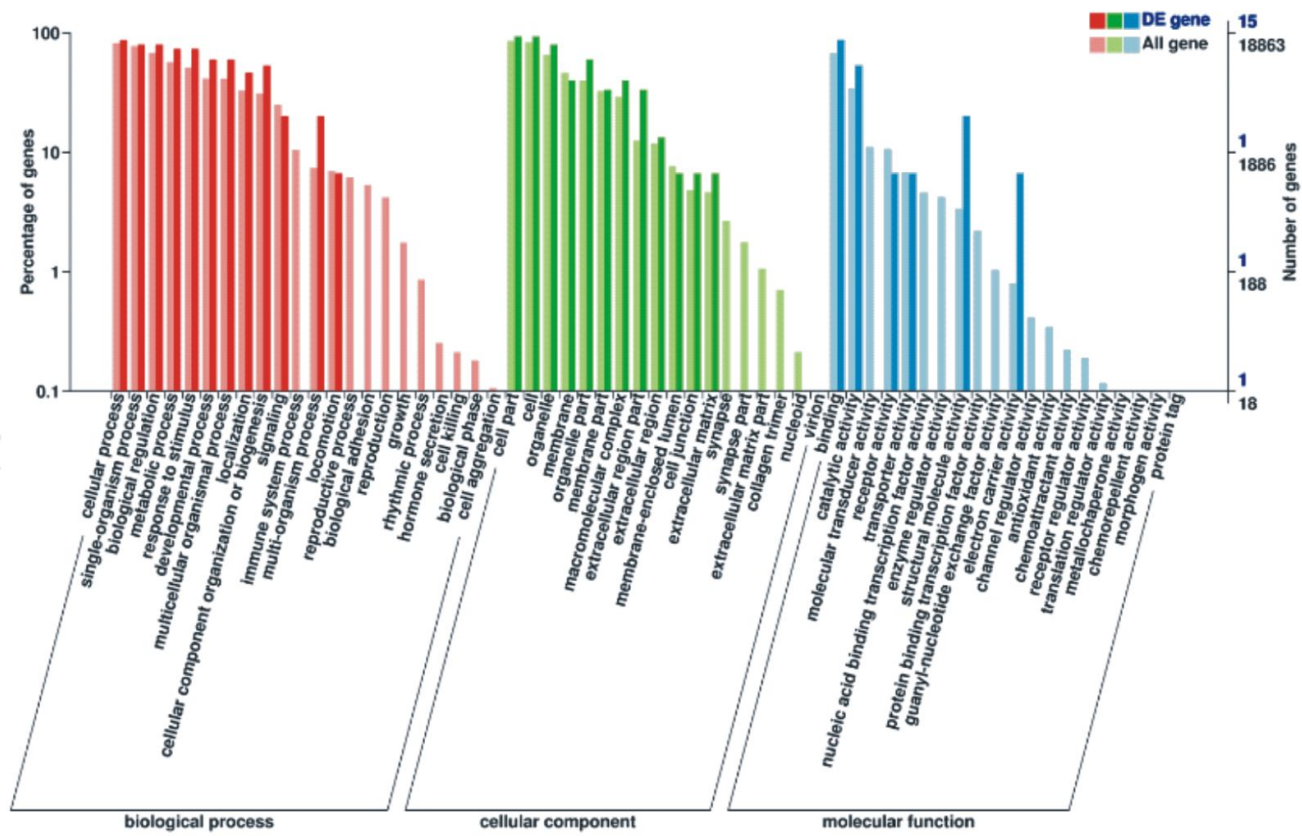

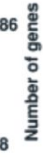

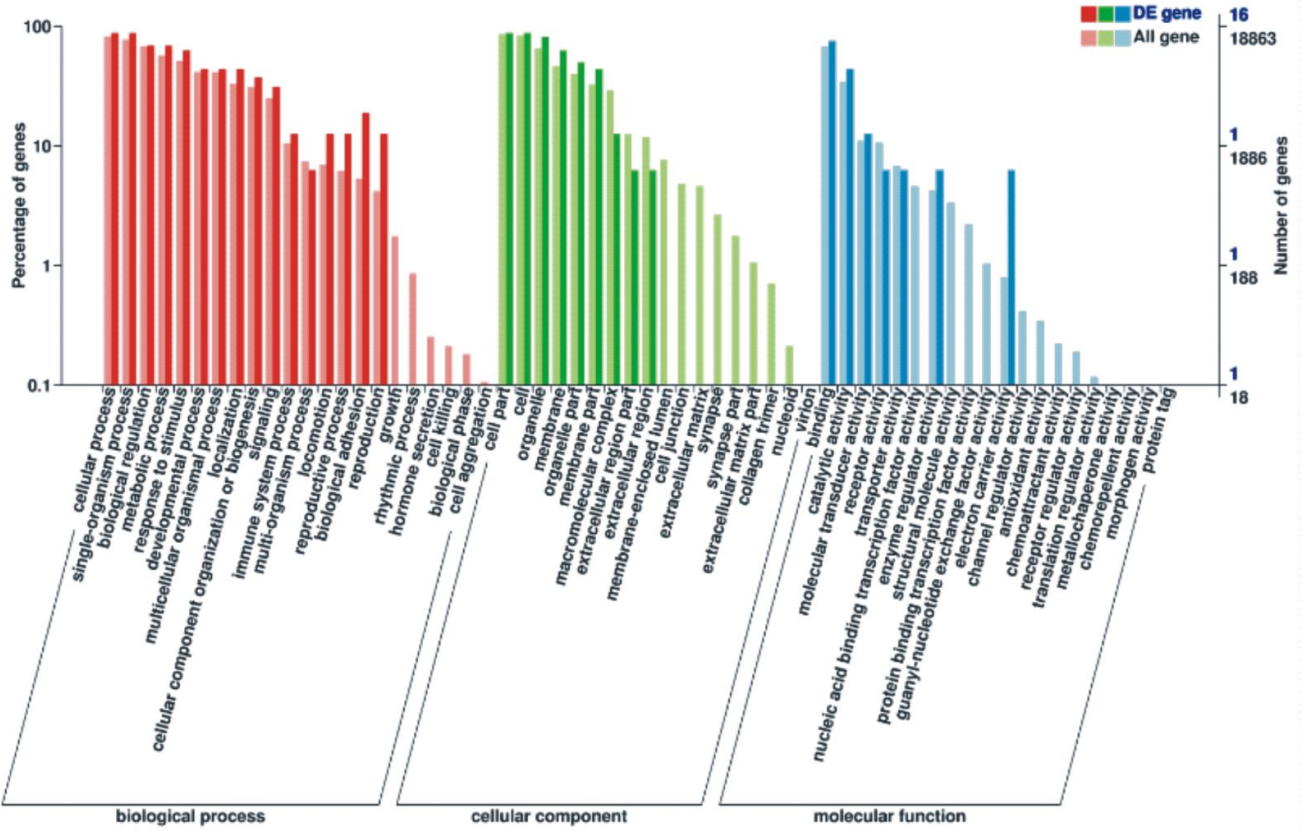

Fig. 4. GO annotation for the differentially expressed genes 
Indian Journal of Animal Health, December, 2018

Transcriptome analysis of the marbling trait

Cluster Dendrogram

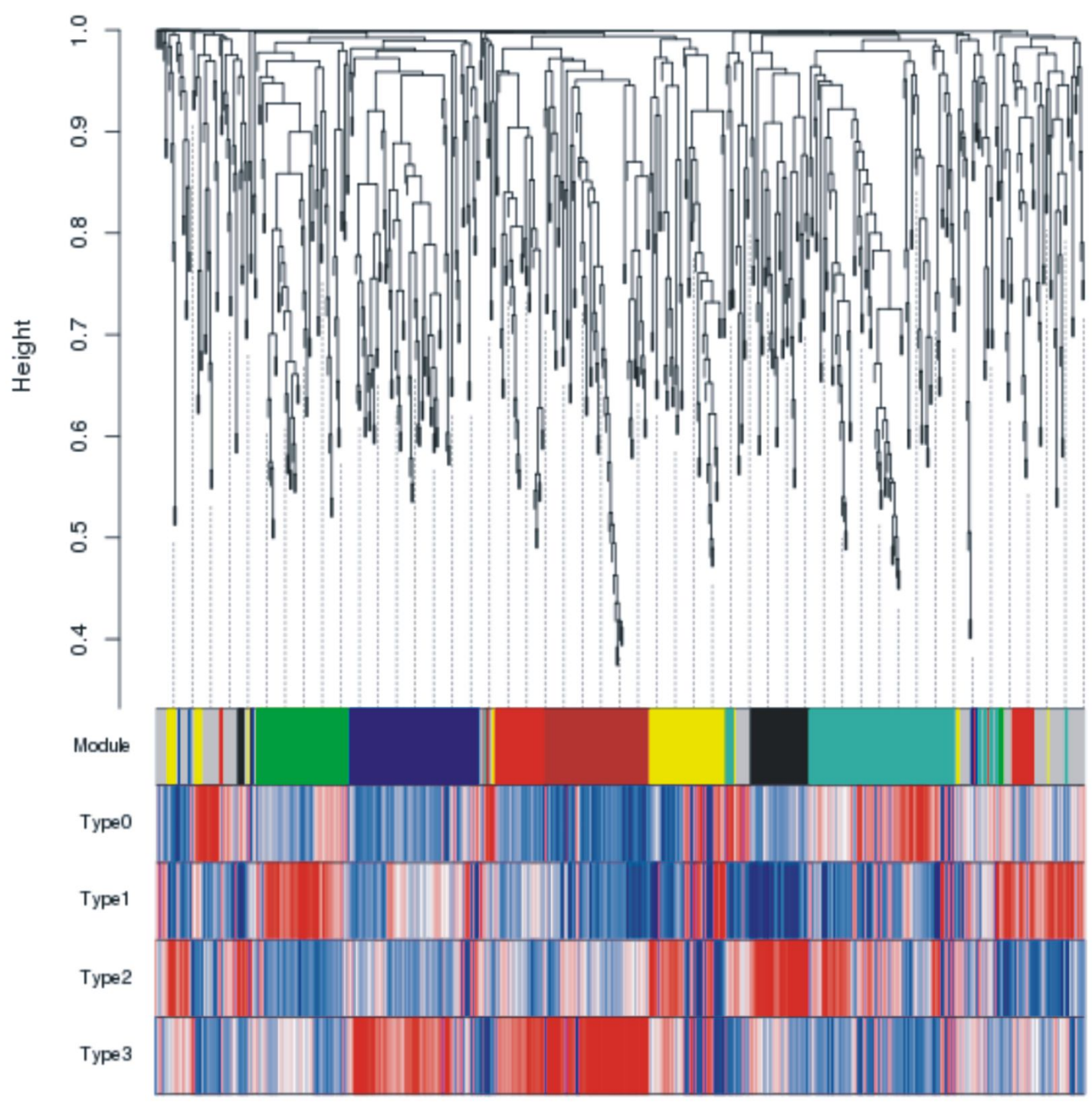

Fig. 5. The cluster tree diagram

(The cluster tree diagram of all genes was analyzed by weighted gene co-expression network analysis and eight gene modules represent different functions, were identified.) 
The protein-coding gene expression level FPKM values was sequenced six orders of magnitude across $10^{\wedge} 2$ to $10^{\wedge} 4$ in the eight samples (Fig. 1).

In addition, the beef ribeye and subcutaneous fat muscle had 15 and 16 differentially expressed genes respectively, with a screening standard of fold change $\geq$ 2 and a false discovery rate $<0.01$. Among them, there were respectively 6 and 14 genes significantly up regulated, and 9 and two genes significantly down regulated (Fig. 2, Fig. 3).

\section{GO functional classification of the} DEGs: We performed GO on the aforementioned DEGs, covering the three domains: cellular component, molecular function and biological processes. Based on the Fisher results $(\mathrm{P}<0.05), 113 \mathrm{GO}$ biological process terms were significantly enriched in beef ribeye (Fig. 3). These included: the regulation of gene expression (GO:0010468) (GO:0010467), the regulation of primary metabolic process (GO:0080090), striated muscle tissue development (GO:0014706), skeletal muscle fiber development (GO:0048741), muscle cell differentiation (GO:0042692) and skeletal muscle thin filament assembly (GO:0030240). In the subcutaneous fat muscle, 157 GO biological process terms were significantly enriched (Fig. 4). These included: the positive regulation of gene expression (GO:0010628) (GO:0010468), regulation of primary metabolic process (GO:0080090), tissue development (GO:0009888), skeletal muscle cell differentiation (GO:0035914) and skeletal muscle satellite cell differentiation (GO:0014816).

Differential expression of gene pathway enrichment: KEGG pathway enrichment analysis for the DEGs can be indicative of the biological implications and possible interactions (Du et al., 2014). Here, the "KEGG Functional Annotation Chart" module in DAVID was used to categorize the DEGs. In beef ribeye, there were 18 KEGG pathways significantly enriched ( $P$ $<0.05)$, including the oxytocin signaling pathway (ko04921) and vascular smooth muscle contraction (ko04270). Of the 19 KEGG pathways enriched in the subcutaneous fat muscle $(\mathrm{P}<0.05)$, the regulation of the actin cytoskeleton (ko04810) and ovarian steroidogenesis (ko04913) were thought to be of particular interest.

\section{DISCUSSION}

This study analyzed the underlying molecular mechanism and complexity of fat deposition and its impact on meat quality at a transcriptional level.

All genes were analyzed by weighted gene co-expression network analysis and eight gene modules were identified (Fig. 5). Following an enrichment analysis to obtain specific KEGG and GO term and binding characters, the blue module was shown to be closely related to lipid metabolism, such as the gene for human Emopamil binding protein that participates in sterol metabolism (Ma et al., 2007). Also, cellular retinoic acid-binding protein 2 , a member of the cytoplasmic/ lipid-binding protein 
family, participates in the vitamin A signaling pathway and increases the low density lipoprotein cholesterol cycle (Yang et al., 2016). Further, some new genes were successfully identified, including gene 2869 and glycerophosphocholine phosphodiesterase 1 , which regulates lipid metabolism in biological processes (GO: 0006629).

A further analysis using the CLC Genomics workbench 6 software packages identified 15 and 16 differentially expressed genes between beef ribeye and subcutaneous fat muscle respectively. In respect to this, 6 and 14 of which were significantly upregulated, and 9 and 2 genes were significantly down-regulated. The expression of the protein-coding genes cytochrome $\mathrm{P} 450$, family 1 , subfamily A, polypeptide 1 (CYP1A1) and Ras-related Associated with Diabetes (RRAD) was significantly increased in muscle fat of high marble level as compared to low marbled meat $(\mid \log 2$-fold change $|=2.09,| \log 2$-fold change $\mid=1.90$ ). Previous studies suggested that cytochrome P450, family 21, subfamily A, polypeptide 2 (CYP21A2) of the cytochrome $\mathrm{P} 450$ family is involved in the synthesis of cholesterol (Wang et al., 2017b), steroids and other lipids, and that CYP1A1 may also be involved in lipid synthesis. The CYP1A family is mainly involved in the metabolism of polycyclic aromatic compounds and aromatic amines. CYP1A1 was activated by intensifying polychlorinated biphenyls to interact with hepatic 7-ethoxyl-isophenoxazole deethylation and testosterone-6- $\beta$ - hydroxylase in the liver and testicles (Machala et al., 1998). Moreover, hepatic triglyceride synthesis involves the reaction of glycerol and ATP. Triglyceride and phosphatides are the main constituents of IMF (Large et al., 2004) and also the testes are closely related to the deposition of intramuscular fat (Wang et al., 2017b). Usually, the mammalian reproductive organs are damaged after castration, leading to lower sex hormone levels and an increased carbohydrate and lipid metabolism, thereby increasing the deposition of intramuscular fat and thus improving the meat quality. RRAD inhibits CAMK2D activation and phosphorylation. In arrhythmia pathways, RRAD has a strong inhibitory effect on the $\mathrm{Ca} 2+$ current of the L-closed circuit and co-regulates its trafficking to the cell membrane. The myocardial hypertrophy can also be inhibited by calcium/ calmodulin dependent kinase II. By comparing T29 and T29H cell lines, researchers found that silencing RRAD by DNA methylation promotes glucose and lactic acid uptake (Wang et al., 2014). The characteristics of lactic acid and fat deposition were positively correlated previously (Duan and Zhou, 2010) and the up regulated genes CYP1A1 and RRAD are, therefore, thought to be involved in the deposition of IMF and the formation of the marble texture.

Similarly, the transcription of the proteincoding gene BCR (KLHL40) was also positively correlated with meat quality. This E3 ubiquitin ligase complex is important for the development of skeletal muscle and 
regulates the activity of the dimerization partner $1(\mathrm{E} 2 \mathrm{~F})$ and dimerization partner (DP1) transcriptional complex by mediating the ubiquitination and subsequent degradation of transcription factor Dp-1. The Kelch-like family member 40 (KHLH40) proteins promotes the stability of leiomodin 3 by negatively regulating its ubiquitination. Interestingly, leiomodin 3 has the ability to microfilament nucleate and is usually enriched at the end of the fibrous filament and expressed during early muscle development (Kreissl, 2014). Moreover, mutations in the KLHL40 gene have been shown to cause congenital nemaline myopathy (Benito et al., 2016). Another up regulated gene in our study encoded for adenosine monophosphate deaminase 3 , an isoform encoded by erythrocytes. Other isoforms of the AMP deaminase gene family are predominantly encoded in muscle and liver cells. In this study, the thyroid hormone response protein (THRSP) was one of the strongest up regulated genes in subcutaneous fat muscle with good marble meat quality. THRSP is known to be expressed in liver and fat cells, especially in lipomata like breast tumors. This suggests that THRSP has a certain role in regulating lipid metabolism and may have a role in tumorigenesis (Cui et al., 2015). Reassuringly, in cattle, chickens, pigs, and sheep, THRSP has already been studied in relation to meat quality. In mice, spot 14 was identified as a THRSP homolog and the in vitro post-transcriptional protein has been used to treat hypothyroidism (Seelig et al., 1981). Spot 14 proteins was found in the nucleus or cytoplasm (Kinlaw et al., 1992) in liver, breast, and white and brown adipose tissues in mice (Jump et al., 1985). Moreover, THRSP expression can increase fat generation enzyme levels, such as fatty acid synthase in liver (Kinlaw et al., 1995). Kirschner and Mariash (1999) found that body index and fasting could trigger a reduction in THRSP mRNA. However, Ortega et al. (2010) found that human THRSP mRNA was negatively correlated with obesity. Nevertheless, THRSP interacts with midline 1 interacting protein 1 to inhibit its interaction with acetyl-CoA carboxylase A. Clearly, THRSP has a role in the biosynthesis of triglycerides and medium fatty acid chains, thereby regulating lipogenesis and important for the deposition of intramuscular fat (Komolka et al., 2014).

The down regulated gene encoding for the CD5 intact protein in beef ribeye has previously been observed in avirulent Th17 cells and can reduce the content of polyunsaturated fatty acyl. This, in turn, promotes the two metabolic proteins methylsterol monooxygenase 1 and CYP51A1 to synthesize ligands of the nuclear receptor ROR-gamma (RORC), ultimately inhibiting RORC activity and inflammatory gene expression. In brief, CD5L regulates the intracellular fat composition and inhibits the cholesterol synthesis (Wang et al., 2015). The downregulation of $\mathrm{CD} 5 \mathrm{~L}$, as observed in this study, is therefore believed to promote the deposition of IMF. Other down regulated genes from our study, such as keratin 18 , regulate cell differentiation and have been 
associated with the liver cell antithrombin complex (Sudano et al., 2014).

In vivo, the biological function of a single protein is not independent, but based on the coordination of a set of genes/proteins. Here, we used the DAVID "GO Functional Annotations Chart" software and found that the GO terms of the DEGs of beef ribeye, as compared to subcutaneous fat muscle, were mostly associated with the development of monomers, myocardial contraction, protein binding and mitochondrial membranes. Among them, myosin light chain 9 (MYL9) and $\alpha-2$ actin (ACTA2) are expressed in striated muscle tissue and participate in muscle cell differentiation and skeletal muscle growth respectively. For the subcutaneous fat muscle, the most relevant GO terms of the upregulated genes included the negative regulation of gene expression, main metabolic pathways and the regulation tissue growth. For example, myosin light chain kinase 4 (MYLK4) is involved in skeletal muscle growth and the differentiation of myocardial cells. The GO terms of the down regulated DEGs, on the other hand, were associated with the differentiation of hematopoietic progenitor cells and the negative regulation of the activational receptor signaling pathway. Different body parts get different GO terms, which may be the reason for the change in IMF content of the different meat textures. For example, the GO terms of beef ribeye samples were mainly enriched in the rhabdomyolysis and mitochondria. The striated muscles are a red muscle, rich in mitochondria and with a high-fat content given that its polyunsaturated fatty acid content is about $6 \%$ higher than white muscle (Zuo, 2014). KEGG analyzed the DEG of the beef ribeye and related them mostly to vascular smooth muscle contraction and oxytocin, systemic lupus erythematosus, adjusting the actin cytoskeleton and the metabolism of arachidonic acid (AA). The latter is an omega- 6 fatty acid that stimulates growth and the different genes through the phosphatidylinositol-3-kinase signaling pathway (Hughes-Fulford et al., 2006). In subcutaneous fat, the DEGs in intramuscular fat were shown to mainly regulate the muscular protein skeleton and purine metabolism, which has some effects on lipids (Wolfram, 1989). Both the beef ribeye and the subcutaneous fat muscle were both enriched in the vascular smooth muscle contraction (ko04270) KEGG pathway, which is associated with muscle development and fat deposition. Our study showed that up regulated DEGs, like myosin light streptokinase, and down regulated DEGs, like myosin light-chain, are likely to be associated with Angus beef muscle. Myosin light streptokinase is a member of the muscle immunoglobulin gene family that regulates the phosphorylation of the regulatory lightchain and plays a role in the smooth muscle cells (Sweeney et al., 1993). Myosin lightchain is a calcium/ calmodulin-dependent enzyme of the muscle immunoglobulin gene family. Taken together, the results obtained from our study showed that the vascular smooth muscle contraction 
(ko04270) pathway could regulate intramuscular fat deposition through myosin and $\mathrm{Ca}_{2+}$ channels.

This study used transcriptome sequencing and further analyses to compare different meat qualities of Angus cattle of beef ribeye and subcutaneous fat muscle using Illumina high-throughput sequencing technologies. Following the comparison analysis, we identified two significantly up regulated differentially expressed genes CYP1A1 and RRAD, the enrichment of the vascular smooth muscle contraction pathway and AA metabolism. By extension, the previous were thought to be related to the mechanisms underlying muscle fat contribution, composition and the distribution of marbled patterns. However, deposits of IMF in the beef ribeye or the subcutaneous fat muscle may also relate to biological activities such as mitochondrial capacity and purine metabolism (Wang et $a l ., 2017 \mathrm{a})$. Previously it was suggested that IMF deposition differences between different breeds of cattle might be related to differences in energy metabolism maintenance and fatty acids synthesis by the liver. In collaboration with our research team, the genes encoding for acetyl-CoA carboxylase $\beta$ and apolipoprotein A1 were found to be key for lipid metabolism (Wang et al., 2017a). In addition, DEGs as a result of castration were shown to be involved in immunity, lipid and fatty acid metabolism and protein synthesis (Wang et al., 2017b). Looking at the candidate genes regulating the marbled meat texture, as identified in the current study, it was concluded that their gene expression may have been determined by a variety of factors including castration and the part of the Angus cattle. In order to improve the Angus cattle meat quality, the cattle industry should breed new varieties as part of healthy and sustainable development. The small sample size is an acknowledged limitation of our study and probably explains the relatively small number of DEGs. A more in-depth analysis of the functions of these DEGs and their association with meat quality will be addressed in future studies.

Competing interests: The authors declare that they have no competing interests.

\section{ACKNOWLEDGEMENTS}

First of all, I would like to extend my sincere gratitude to my tutors, X.W. and W.L., for their instructive advice and useful suggestions on my thesis. I am deeply grateful of their help in the completion of this thesis. This work was supported by the Scientific and Technological Project in Shanxi Province (201703D221021-1) and Breeding Project in Shanxi Academy of Agricultural Sciences (17YIgc109). I am also deeply indebted to all the other teachers in Translation Studies for their direct and indirect help to me. Special thanks should go to my friends who have put considerable time and effort into their comments on the draft. Finally, I am indebted to my parents for their continuous support and encouragement. 


\section{REFERENCES}

Aksyonov SA, Bittner M, Bloom LB, RehaKrantz LJ and Gould IR et al., 2006. Multiplexed DNA sequencing by synthesis. Anal Biochem, 348: 127-138

Benito DN, Nascimento A, Abicht A, Ortez C and Jou C et al., 2016. KLHL40-related nemaline myopathy with a sustained, positive response to treatment with acetylcholinesterase inhibitors. J Neurol, 2016 (263-3): 517-523

Blencowe BJ, Ahmad S and Lee LJ, 2009. Current-generation high-throughput sequencing: deepening insights into mammalian transcriptomes. Genes Dev, 23: 1379-1386

Chen D, 2015. Transcriptome study on the molecular mechanism of the different marbling phenotype in beef cattle. Doctoral Dissertation, China Agricultural University, Beijing, China

Cui YJ, Liu ZY, Sun X, Hou XM and Qu B et al., 2015. Thyroid hormone responsive protein spot 14 enhances lipogenesis in bovine mammary epithelial cells. In Vitro Cell Dev Biol Anim, 51(6): 586-594

Du C, Fu S, Gao HY, Zhang ZQ and Meng XR et al., 2014. Transcriptome analysis of intramuscular preadipocytes and mature adipocyte in Cashmere goats. CJAVS, 45: 714-721

Duan Y and Zhou L, 2010. Correlation of muscle glycolytic potential and its components with meat quality, fat deposition and blood indexes in pigs. Acta Agriculturae Universitatis Jiangxiensis, 32(6): 1224-1229
Grobet L, Martin LJ, Poncelet D, Pirottin D and Brouwers B et al., 1997. A deletion in the bovine myostatin gene causes the doublemuscled phenotype in cattle. Nat Genet, 17: 71-74

Hou DX, 2011. Study on the role of free fatty acids in insulin resistance in adipose tissue and the application of Solexa in miRNAs detection. Doctoral Dissertation, Nanjing University, Jiangsu, China

Hughes-Fulford M, Li CF, Boonyaratanakornkit J and Sayyah S, 2006. Arachidonic acid activates phosphatidylinositol 3kinase signaling and induces gene expression in prostate cancer. Cancer Res, 66(3): 1427

Hwang Y, Kim G, Jeong J, Hur S and Joo S, 2010. The relationship between muscle fiber characteristics and meat quality traits of highly marbled Hanwoo (Korean native cattle) steers. Meat Sci, 86: 456-461

Jump DB and Oppenheimer JH, 1985. High basal expression and 3,5,3'triiodothyronine regulation of messenger ribonucleic acid S14 in lipogenic tissues. Endocrinology, 117(6): 2259-2266

Kinlaw WB, Church JL, Harmon J and Mariash $\mathrm{CN}, 1995$. Direct evidence for a role of the "spot 14" protein in the regulation of lipid synthesis. J Bio Chem, 270 (28): 16615-16618

Kinlaw WB, Tron P and Friedmann AS, 1992. Nuclear localization and hepatic zonation of rat "spot 14" protein: immunohistochemical investigation employing 
anti-fusion protein antibodies. Endocrinology, 131(6): 3120-3122

Kirschner LS and Mariash CN, 1999. Adipose S14 mRNA is abnormally regulated in obese subjects. J Ame Thy Ass, 9(2): 143-148

Komolka K, Albrecht E and Kühn C, 2014. Expression of thyroid hormoneresponsive protein (THRSP) is related to intramuscular fat in a F2-cross between Charolais and Holstein. Aso Lat Pro Ani, 22: 618-621

Kreissl M, 2014. Molecular mechanism of muscle weakness in congenital myopathy caused by mutations in TPM3 and LMOD3. Postgraduate Dissertation, University of Sydney, New South Wales, Australia

Large V, Peroni O, Letexier D, Ray H and Beylot M et al., 2004. Metabolism of lipids in human white adipocyte. Diabetes Metab, 30(4): 294-309

Li B, 2002. The adaptation of Angus bull in Canada is reported in Guizhou province. China herbivores, 22: 15-17

Li P, Piao Y, Shon H and Ryu K, 2015. Comparing the normalization methods for the differential analysis of Illumina highthroughput RNA-Seq data. BMC Bio, 16: 347

Ma J, Dempsey AA, Stamatiou D, Wayne K and Marshall W et al., 2007. Identifying leukocyte gene expression patterns associated with plasma lipid levels in human subjects. Atherosclerosis, 191: 6372

Machala M, Neèa J, Drábek P, Ulrich R and
Sabatová V et al., 1998. Effects of chronic exposure to PCBs on cytochrome P450 systems and steroidogenesis in liver and testis of bulls (Bos taurus). Comp Biochem Physiol A Mol Integr Physiol, 120(1): 65-70

Ortega FJ, Martin AV, Navarrete JM, Bassols J and Hermosa JR et al., 2010. Thyroid hormone responsive Spot 14 increases during differentiation of human adipocytes and its expression is downregulated in obese subjects. Int $\mathrm{J}$ Obes, 34(3): 487-499

Pethick DW, Harper GS and Oddy VH, 2004. Growth development and nutritional manipulation of marbling in cattle. Anim Prod Sci, 44: 705-715

Scollan N, Hocquette J, Nuernberg K, Dannenberger D and Richardson I et al., 2006. Innovations in beef production systems that enhance the nutritional and health value of beef lipids and their relationship with meat quality. Meat Sci, 74: 17-33

Seelig S, Liaw CW and Towh HC, 1981. Thyroid hormone attenuates and augments hepatic gene expression at a pretranslational level. Proceedings of the National Academy of Sciences of the United States of America, 78(8): 47334737

Sudano MJ, Caixeta ES, Paschoal DM, Martins $\mathrm{A}$ and Machado $\mathrm{R}$ et al., 2014. Cryotolerance and global gene-expression patterns of Bos taurus indicus and Bos taurus taurus in vitro and in vivo produced blastocysts. Reprod Fertil Dev, 26(8): 1129-1141 
Sweeney HL, Bowman BF and Stull JT, 1993. Myosin light chain phosphorylation in vertebrate striated muscle: regulation and function. Am J Phy, 264(1): 1085-1095

Wang C, Yosef N, Gaublomme J, Wu C and Lee YJ et al., 2015. CD5L/AIM Regulates Lipid Biosynthesis and Restrains Th17 Cell Pathogenicity. Cell, 163(6): 14131427

Wang X, Zhang YQ, Zhang XZ, Wang DC and Jin $\mathrm{G}$ et al., 2017a. The comprehensive liver transcriptome of two cattle breeds with different intramuscular fat content. Biochem Biophys Res Commun, 490(3): 1018-1025

Wang X, Zhang YQ, Zhang XZ, Wang DC and Jin Get al., 2017b. Transcriptome analysis of castrated bovine reveals the characters of protein accumulation. Indian J Anim Res, 2017, 51 (OF)
Wang Y, Li G, Mao F, Li X and Liu Q et al., 2014. Ras-induced epigenetic inactivation of the RRAD (Ras-related associated with diabetes) gene promotes glucose uptake in a human ovarian cancer model. J Biol Chem, 289(20): 14225-14238

Wolfram MG, 1989. Genetic and therapeutic aspects of lipid and purine metabolism. Springer, Berlin Heidelberg, Germany, 147

Yang QY, Wang R, Xiao WF, Sun FY and Yuan $\mathrm{H}$ et al., 2016. Cellular retinoic acid binding protein 2 is strikingly down regulated in human esophageal squamous cell carcinoma and functions as a tumor suppressor. PloS One, 11(2): e0148381

Zuo X, 2014. Introduction the problems and solution in cattle breeding. Hans J Agr Sci, 12: 1 